\title{
Automated Car Number Plate Detection System to detect far number plates
}

\author{
Anisha goyal ${ }^{1}$, Rekha Bhatia ${ }^{2}$ \\ ${ }^{1}$ (Department of CSE ,Punjabi University Regional Centre for Information Technology and Management, \\ Mohali, Punjab,India) \\ ${ }^{2}$ (Department of CSE ,Punjabi University Regional Centre for Information Technology and Management, \\ Mohali, Punjab,India)
}

\begin{abstract}
The Automatic number plate recognition (ANPR) is a mass reconnaissance strategy that uses optical character recognition on pictures to peruse the tags on vehicles. They can use existing shut circuit TV or street standard enforcement cameras, or ones particularly designed for the errand. They are used by different police forces and as a technique for electronic toll collection on pay-per-use streets and monitoring movement action, for example, red light adherence in an intersection. ANPR can be used to store the pictures caught by the cameras and in addition the content from the tag with some configurable to store a photo of the driver. Systems ordinarily use infrared lighting to permit the camera to take the photo whenever of the day. A capable blaze is included in no less than one form of the intersection monitoring cameras, serving both to illuminate the photo and to make the offender mindful of his or her slip-up. ANPR innovation tends to be district particular, owing to plate variety from spot to put. A different recognition strategies have been produced and number plate recognition systems are today used in different movement and security applications, for example, parking, access and border control, or tracking of stolen autos. Till now, all the LPR systems have been created using neural networks. This work proposes to execute the system using Gabor filter, OCR and Vision Assistant to make the system quicker and more proficient.
\end{abstract}

Keywords: ANPR, Localization, segmentation, recognition, Optical Character Recognition (OCR)

\section{Introduction}

ANPR is an image-processing innovation which is used to perceive vehicles by their tags. This expertise is ahead of time ubiquity in security and traffic installation. Tag Recognition System is an application of PC vision. PC vision is a technique for using a PC to take out abnormal state information from a digital image. The useless homogeny among various tags for example, its dimension and the outline of the License Plate.

The ANPR system consists of following steps:-

i. Vehicle image capture.

ii. Preprocessing.

iii. Number plate extraction.

iv. Character segmentation.

v. Character recognition.

The ANPR system works in these strides, the initial step is the location of the vehicle and capturing a vehicle image of front or back perspective of the vehicle, the second step is the localization of Number Plate and then extraction of vehicle Number Plate is an image. The final stride use image segmentation strategy, for the segmentation a few techniques neural network, mathematical morphology, color analysis and histogram analysis. Segmentation is for individual character recognition. Optical Character Recognition (OCR) is one of the strategies to perceive the every character with the assistance of database stored for separate alphanumeric character.

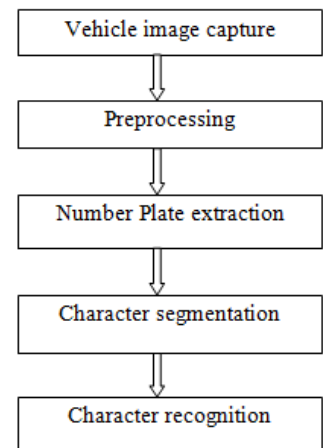

Fig1.1:- Block diagram of ANPR system 


\section{Literature Review}

Muhammad Tahir Qadri In this anticipate [2] for the recognition the OCR techniques is used which is susceptible to misalignment and to various sizes. The affine transformation can be used to advance the OCR recognition from various size and angles. The programmed vehicle identification system using vehicle license plate is exhibited. A series of image processing techniques of the system for identifying the vehicle from the database stored in the PC.

S.Kranthi, K.Pranathi In this paper they [3] proposed that Automatic Number Plate Recognition (ANPR) is a method that catches the vehicle image and confirmed their license number. ANPR can be used in the presentation of stolen vehicles. ANPR can be used in various manners by using to identify it stolen vehicle on the highway.

Abd KadirMahamad In this paper they explained [8] an automatic number plate inspection of letter sets of plate using image processing and optical character recognition. An imperative system has been created of training interface using LABVIEW software.

Kuldeepak et al. In this paper [1] they introduced that high level of precision has been required by the number plate recognition when streets are occupied and number of vehicles are passing through. In this paper, by optimizing different parameters, they have accomplished an exactness of $98 \%$. It is essential that for the tracking stolen vehicles and monitoring of vehicles of an exactness of $100 \%$ can't be bargained with. Therefore to accomplish better precision streamlining is required. Additionally, the issues like stains, blurred regions, smudges with various text style and sizes ought to be remembered. This work can be further boundless to minimize the errors because of them.

AmrBadr et al. In this paper [8] Automatic recognition of car license plate number got to be indispensible part in our day by day life. This paper mainly explains an Automatic Number Plate Recognition System (ANPR) using Morphological operations, Histogram manipulation and Edge discovery Techniques for plate localization and characters segmentation. Artificial Neural Networks are used for Character classification and recognition.

\section{Anpr System Model}

Typical ANPR system consists of four steps these are Image Acquisition, License Plate extraction, character segmentation, and character recognition.

\subsection{Image Acquisition}

The initial step is the Acquisition of an image i.e., getting an image using the digital camera associated with the PC. These Caught images are in RGB format so it can be further process for the Number Plate Extraction. The database system contains the personal information of the vehicle proprietor and a few plate vehicle images, abbreviations and acronyms.

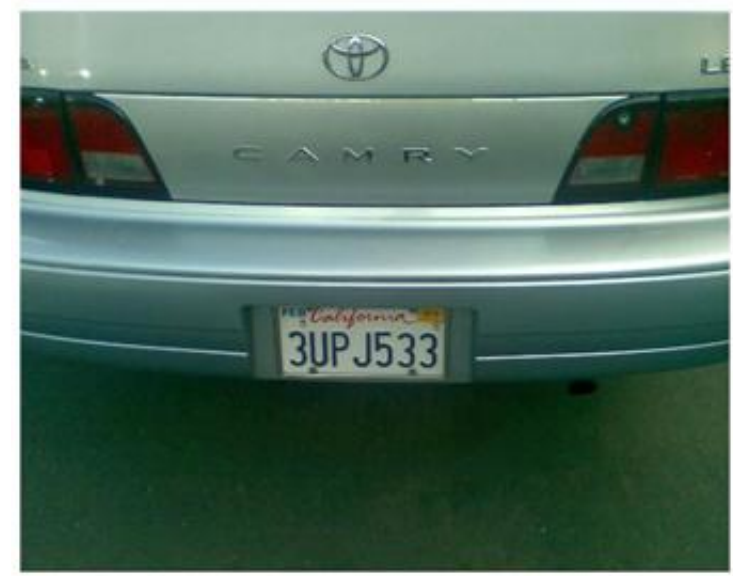

Fig3.1:- Captured image by digital camera

\subsection{Image Processing}

Captured RGB image is appeared in fig2.The captured image is influenced by many elements like: Optical system distortion, system commotion, lack of presentation or over the top relative motion of camera or vehicle and so forth result is the degradation of a captured vehicle image and the unfriendly influence to the further image processing. Therefore before the main image processing, pre-processing of the captured image ought to be taken out which include converting RGB to gray in fig 3, clamor evacuation, and border enhancement for brightness. 


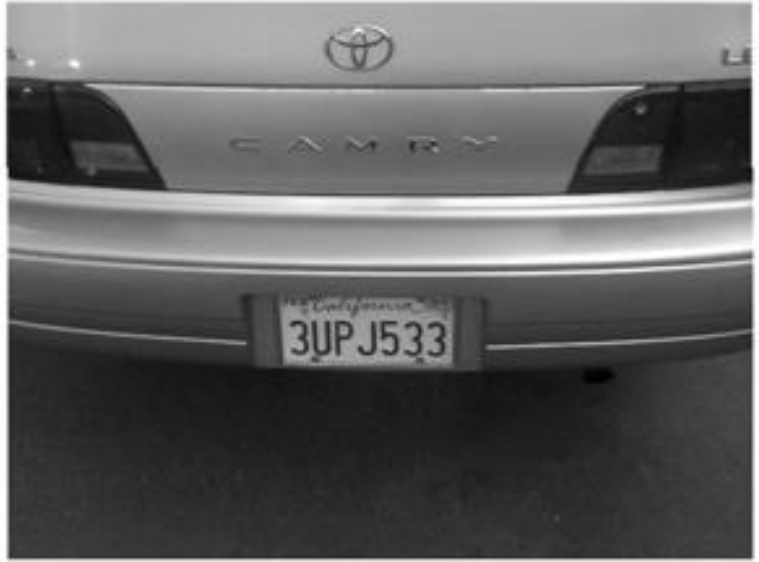

Fig3.2:- converted from color image to gray image

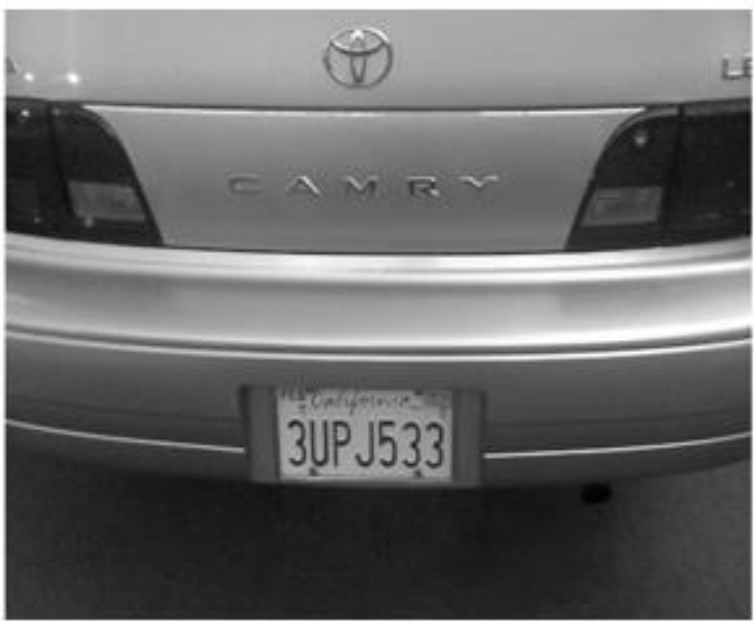

Fig3.2: - Pre-Processed image

\subsection{Plate Localization}

The Basic stride in recognition of vehicle Number Plate is to identify the plate size. By and large number plates are rectangular plate mat lab toolbox function gives a function called region props. It quantifies a set of properties for each marked region in the matrix. We used bounding box to gauge the properties of the image region. In the wake of labeling the associated components, the region will extricate from the input image. Number plate localization is appeared in figure.

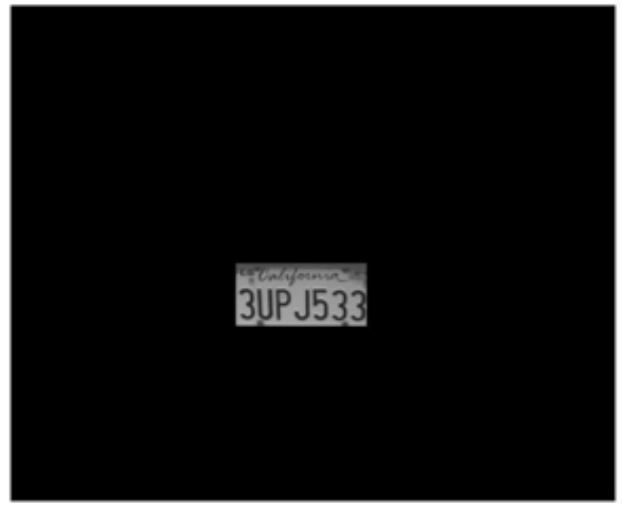

Fig3.3:- Vehicle Number Plate Localization

\subsection{Plate Segmentation}

Number plate segmentation assumes an imperative role in ANPR system. The essential thought after region growing is to remember one or more criteria that are quality for the wanted region. Subsequent to 
establishing the criteria, the image is looked for any pixels that satisfy the necessities. At whatever point such a pixel is experienced, its neighbors are checked, and if any of the neighbors likewise parallel the criteria, both the pixels are measured as have a place with the same region. We get individual character and number image by using, vertical and horizontal scanning technique. Fig demonstrates the plate segmentation case.

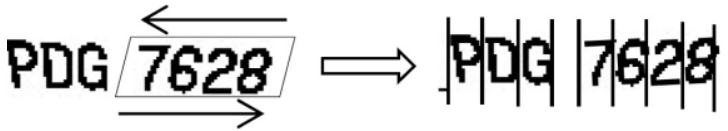

Fig3.4-: Example of Plate Segmentation.

\subsection{Character Recognition}

This is the most essential and basic phase of the ANPR system. It displays the techniques that were used to order and then perceive the individual characters. The classification is based on the extracted features. These features are then arranged using either the statistical, syntactic or neural methodologies.

Distinctive strategies were used for character recognition, letters and characters in the paper. Finish the identification by calculating the likeness of features. For the comparable characters, make the second identification with the technique for highlight point matching Another methodology is that Once the lines in an extracted vehicle number plate are separated, the line separation procedure is presently connected segment savvy so individual character can be separated. The separated individual characters are then stored in separate variables. The extracted characters taken from number plate and the characters on database which we have stored are presently coordinated. The following phase is template matching. Template matching is a proficient algorithm for character recognition. The characters image is match up to our given database and the best resembling is considered. Another technique for character recognition is the optical character recognition (OCR) is used to look at the every individual character against the complete alphanumeric database. The OCR really uses relationship strategy to match individual character and finally the number is recognized and stored in string format in a variable. The character is then contrasted and the database for the vehicle authorization. The resultant signs are offered according to the consequence of comparison. Templates will exist for every one of the characters i.e. A-Z and 0-9 as appeared in figure.

\section{ABCDEFGHUJKL MNOPRSTUVYZ 0123456789 \\ Fig3.5-: Database of templates.}

\section{Proposed Methodology}

To recognize number plate first of all add templates from A-Z and 0-9 and add them into mat file. After that read the image and change over that image into gray scale. Presently the following stride is to find out threshold estimation of the image. In the wake of finding T-esteem change over that image into binary.

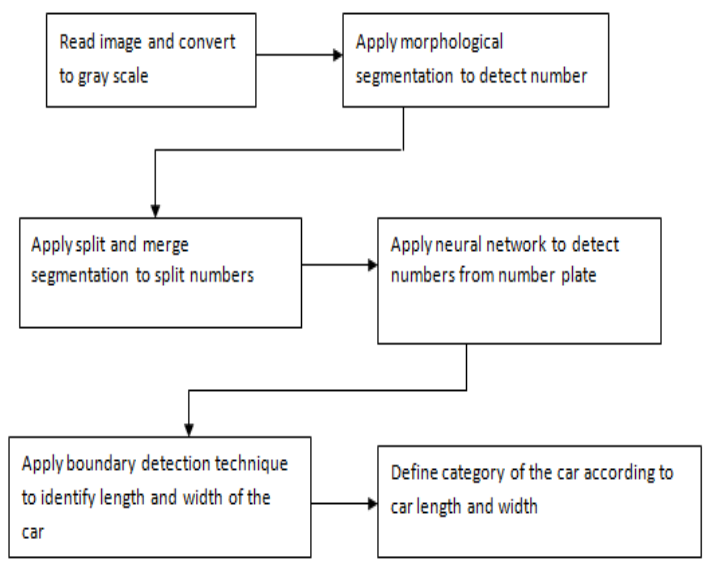

Fig.4.1: Flowchart

As shown in the figure, the block diagram of the proposed procedure is appeared. In the proposed outline the input image is changed over to gray scale and on the gray scale image procedure of morphological scanning is actualized which will check the image and identify number plate part from the auto. The 
distinguished number plate will be given as input to split segmentation which will split every single character and each character will be coordinated with the existing dataset. The most extreme coordinated will identified which will be merged to create final result.

\section{Experimental Results}

The whole scenario has been implemented on MATLAB.

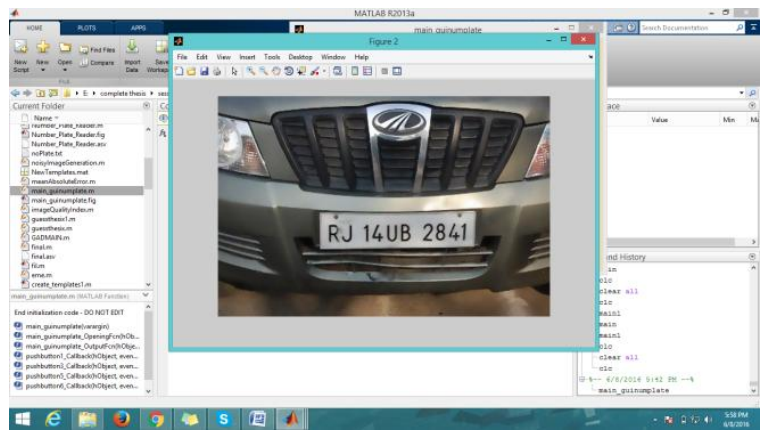

Fig.5.1: Car input for number plate detection

As shown in the figure 5.1, the interface is designed which will detect the number plate from the car and also detect car body. The car image is given as input to detect number plate from the car.

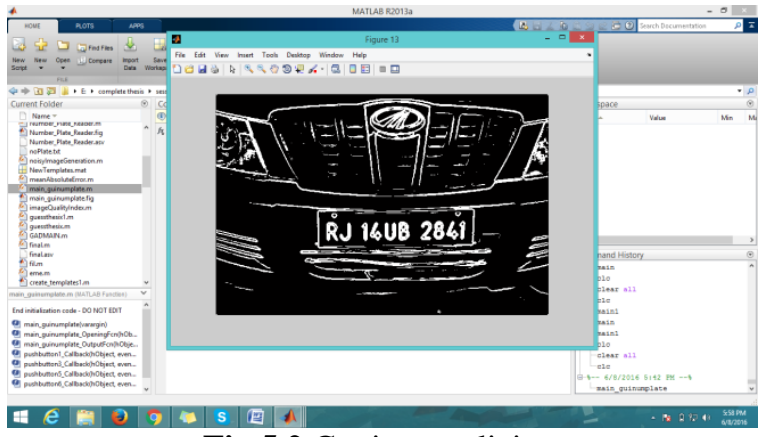

Fig.5.2 Car image slicing

As shown in the figure 5.2, the interface is designed which will detect the number plate from the car and also detect car body. The car image is given as input to detect number plate from the car. The car image is converted into gary scale to recognize number plate. The gray scaleimage is sliced into many parts and each part will be treated independently. The slicing is applied to detect number plate area from the image.

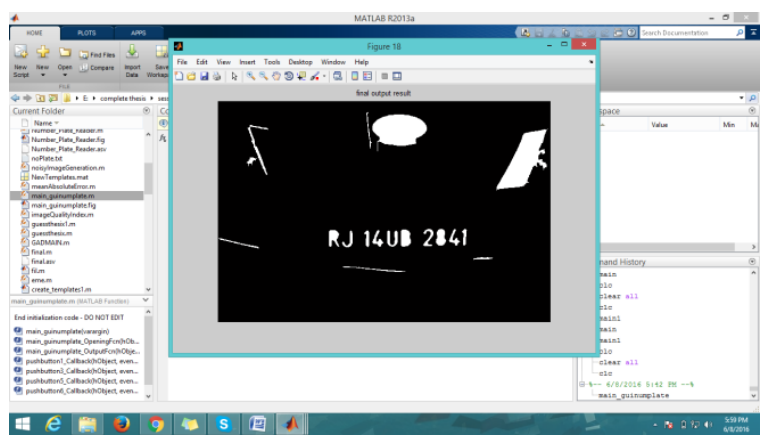

Fig. 5.3 Number Plate Detection

As shown in the figure 5.3, the interface is designed which will detect the number plate from the car and also detect car body. The car image is given as input to detect number plate from the car . The car image is converted into gary scale to recognize number plate. The gray scaleimage is sliced into many parts and each part will be treated independently. The slicing is applied to detect number plate area from the image. When the car number plate is detected, the number plate is recognized from the car image. 


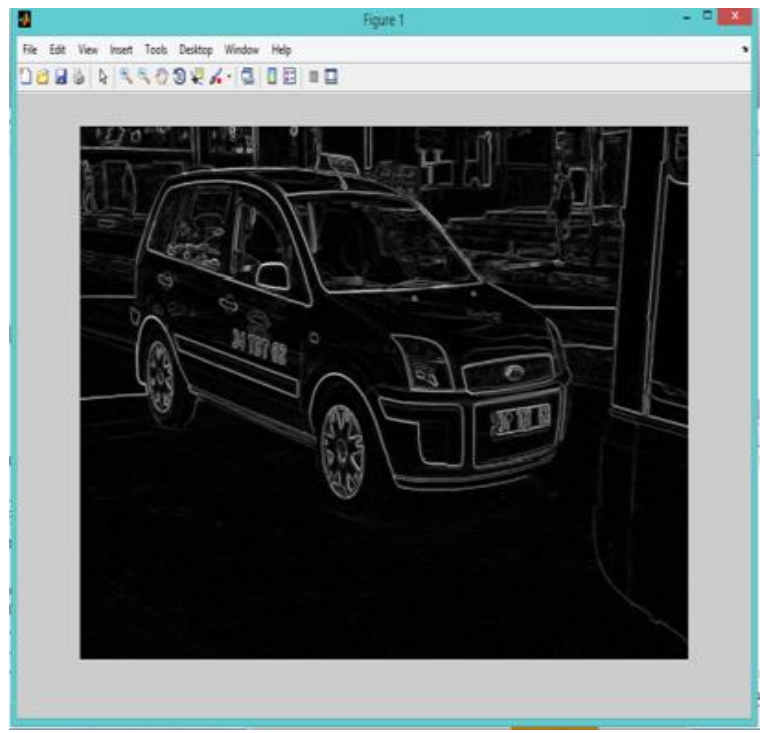

Fig. 5.4 Car image for body Detection

As shown in figure 5.4, the image is given as input to detect the car body. The image which is given as input will be converted into gray scale.

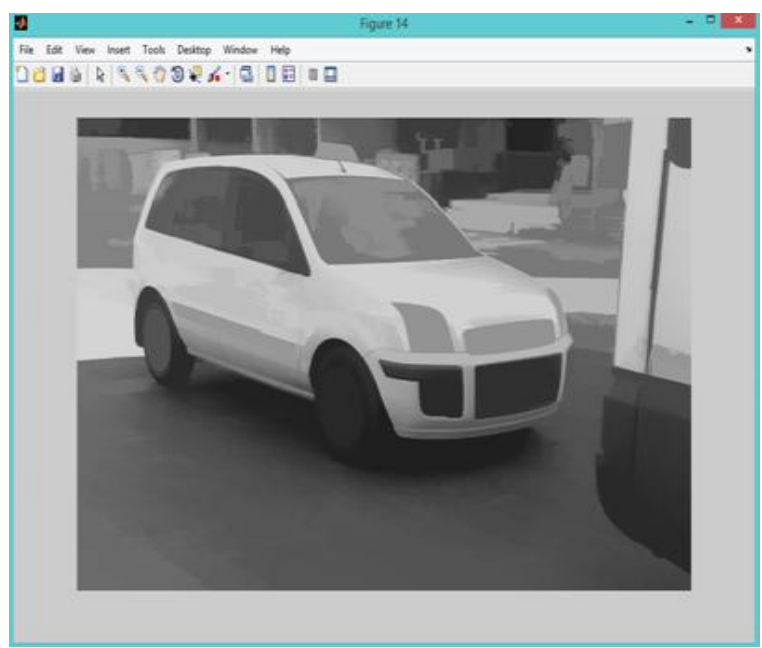

Fig.5.5: Detection of car body

As shown in figure 5.5, the image is given as input to detect the car body. The image which is given as input will be converted into gray scale. The body of the car is detected and car body is marked with white color and other part with the black color.

\section{Conclusion}

In this paper, the automatic number plate recognition system using vehicle license plate is introduced. The system utilizes image processing techniques for recognizing the vehicle from the database stored in the computer by user. The system works agreeably for wide variation of conditions and distinctive sorts of number plates. The system is actualized and executed in Matlab and performance is tried on genuine images. In the existing work, work has been done on contorted number plates. This method has an issue of commotion and image is taken from separation. In proposed work a novel system has been proposed for denoising and for the better character recoginization using standard classifiers of neural networks and give better body detection.

\section{References}

[1]. Kuldeepak, Monika kaushik and Munish Vashishath (2012), "License Plate Recognition System based on Image Processing Using Labview" International Journal of Electronics Communication and Computer Technology (IJECCT) Volume 2 Issue 4 (July 2012).

[2]. Muhammad Tahir Qadri, Muhammad Asif, "Automatic Number Plate Recoginization System For Vechile Identification Using Optical Character Recoginization" 2009 International Conference on Education Technology and Computer. 
[3]. Amar Badr Mohamed M. Abdelwahab, Ahmed M. Thabet, and Ahmed M.Abdelsadek, "Automatic Number Plate Recognition System", Annals of the University of Craiova, Mathematics and Computer Science Series Volume 38(1), 2011, Pages 62\{71ISSN: 1223-6934.

[4]. Markus Friedrich Prokop Jehlicka Johannes Schlaich, "AUTOMATIC NUMBER PLATE RECOGNITION FOR THE OBSERVANCE OF TRAVEL BEHAVIOUR” 8th International Conference on Survey Methods in Transport, France, May 25-31, 2008.

[5]. Nasrul Humaimi Mahmood, Noraishikin Zulkarnain, Nor Saradatul Akmar Zulkifli (2012) International Journal of Engineering Research and Applications (IJERA) ISSN: 2248-9622 www.ijera.com Vol. 2, Issue 3, May-Jun 2012, pp.691-694.

[6]. R. Yusnita, Fariza Norbaya, and Norazwinawati Basharuddin (2012), "Intelligent Parking Space Detection System Based on Image Processing” International Journal of Innovation, Management and Technology, Vol. 3, No. 3, June 2012.

[7]. KazukiMaeno, Hajime Nagahara, Atsushi Shimada, and Rin-ichiro Taniguchi (2013) "Light Field Distortion Feature for Transparent Object Recognition”, Computer vision foundation, IEEE explore, 2013, pp 2786-2793.

[8]. Abd Kadir Mahamad, Sharifah Saon, and Sarah Nurul Oyun Abdul Aziz, "A Simplified Malaysian Vehicle Plate Number Recognition", Springer International Publishing Switzerland 2014

[9]. Rowayda A. Sadek, "SVD Based Image Processing Applications: State of The Art, Contributions and Research Challenges", (IJACSA) ,International Journal of Advanced Computer Science and Applications, Vol. 3, No. 7, 2012.

[10]. Quraishi, M.I.; Choudhury, J.P.; De, M., "Image recognition and processing using Artificial Neural Network," Recent Advances in Information Technology (RAIT), 2012 1st International Conference on, vol., no., pp.95,100, 15-17 March 2012.

[11]. M. Egmont-Petersen, "Image processing with neural networks", Volume 35, Issue 10, October 2002, Pages $2279-23012002$.

[12]. Laxman Poudal, Bhola Thapa, Bim “Application of Digital Image Processing for Shap eCharacterization of Sand Particles", 2011

[13]. Mu-Liang Wang Yi-Hua Liu Bin-Yih Liao, Yi-Sin Lin, Mong-Fong Horng, "A Vehicle License Plate Recognition System Based on Spatial/Frequency Domain Filtering and Neural Networks", Computational Collective Intelligence. Technologies and Applications Volume 6423 of the series Lecture Notes in Computer Science pp 63-70.

[14]. V. Himani et.al, "Automatic Vehicle Number Plate Localization using Symmetric Wavelets", ICT and Critical Infrastructure: Proceedings of the 48th Annual Convention of Computer Society of India, Volume 248 of the series Advances in Intelligent Systems and Computing pp 69-76, 2014.

[15]. Mr. G. T. Sutar1 ,Prof. Mr. A.V. Shah, "Number Plate Recognition Using an Improved Segmentation”, International Journal of Innovative Research in Science, Engineering and Technology (An ISO 3297: 2007 Certified Organization) Vol. 3, Issue 5, May 2014.

[16]. Hanit Karwal, Akshay Girdhar, "Vehicle Number Plate Detection System for Indian Vehicles", IEEE, 2015

[17]. S. Ramalingam, M. Rhead, R. Gurney, "Impact of Character Spacing on the Performance of Automatic Number Plate Recognition (ANPR) Systems through Simulation”, IEEE, 2014 